\title{
TRANSMISSION EXPÉRIMENTALE DU VIRUS DE LA FIÈVRE HÉMORRAGIQUE de Crimée-Congo : PLACe de trols espèces Vectrices DANS LES CYCLES DE MAINTENANCE ET DE TRANSMISSION AU SÉNÉGAL
}

\author{
FAYE O.*,**, CORNET J.P.**, CAMICAS J.L.**, ${ }^{*}$, FONTENILLE D.** ${ }^{* *} \&$ GONZALEZ J.P.***, 1
}

Summary : EXPERIMENTAL TRANSMISSION OF CRIMEAN-CONGO HAEMORRHAGIC FEVER VIRUS: ROLE OF THREE VECTORIAL SPECIES IN MAINTENANCE AND TRANSMISSION CYCLES IN SENEGAL

In this article, we published the role of three species of ticks Amblyomma variegatum (Fabricius, 1974), Hyalomma marginatum rufipes (Koch, 1844) and Hyalomma truncatum (Koch, 1844) in the maintenance and transmission of the CCHF virus. The imagos of these species were infected by intracœlomic route. Vertical transmission (transtasial and transovarial) and horizontal transmission for different stases were studied by isolation on newborn mice, polymerase chain reaction, indirect immunofluorescence and ELISA.

Our results proved that 15 days after inoculation, infection rates of $100 \%$ were noted with Hyalomma marginatum rufipes and Hyalomma truncatum. This rate is about $60 \%$ for Amblyomma variegatum. The imagos of the three species infected have transmitted the virus to their host during blood feeding (100\%). A high transovarial transmission for Hyalomma marginatum rufipes and Hyalomma truncatum were observed (respectively 53 and $50 \%$ ). This rate is about $12 \%$ for Amblyomma variegatum. The tick infection does not persist up to the first generation for the three species studied. Ticks are temporary reservoirs vectors but not permanent reservoirs of CCHF virus.

KEY WORDS : CCHF, transmission, tick, vector, reservoir, Senegal

\begin{abstract}
Résumé :
Dans cet article, nous rapportons la place de trois espèces de tiques Amblyomma variegatum (Fabricius, 1794), Hyalomma marginatum rufipes (Koch, 1844) et Hyalomma truncatum (Koch, 1844) dans les cycles de maintenance et de transmission du virus de la fièvre hémorragique de Crimée-Congo (CCHF) au Sénégal. Les imagos de ces espèces ont été infectés par inoculation intracoelomique. La réplication du virus, les transmissions verticale (transovarienne et transtasiale) et horizontale pour les différentes stases ont été étudiées par les techniques d'isolement sur souriceaux nouveau-nés, d'immunofluorescence indirecte, par la réaction de polymérisation en chaine et par ELISA.

Les résultats obtenus ont montré que 15 jours après l'inoculation, des taux d'infection de $100 \%$ ont été obtenus avec Hyalomma marginatum rufipes et Hyalomma truncatum. Ce taux passe à $60 \%$ chez Amblyomma variegatum. Les imagos des trois espèces infectées ont transmis le virus à leurs hôtes au cours du repas sanguin (100\%). Une transmission transovarienne élevée pour les espèces Hyalomma marginatum rufipes et Hyalomma truncatum a été observée avec respectivement 53 et $50 \%$ de larves positives en immunofluorescence indirecte. Ce taux passe à $12 \%$ chez Amblyomma variegatum. L'infection des tiques ne persiste pas audelà de la première génération chez les trois espèces étudiées. Les tiques sont donc des vecteurs-réservoirs temporaires mais pas des réservoirs permanents du virus CCHF
\end{abstract}

MOTS CLÉS : CCHF, transmission, tique, vecteur, réservoir, Sénégal.

\section{INTRODUCTION}

L e virus de la fièvre hémorragique de CriméeCongo (CCHF) est un arbovirus du genre Nairovirus de la famille des Bunyaviridae pathogène pour l'homme et transmis par les tiques. La distribu-

* Université Cheikh Anta Diop, Dakar, Sénégal.

** Laboratoire ORSTOM de Zoologie Médicale, Institut Pasteur de Dakar, Sénégal.

*** Institut Français de Recherche Scientifique pour le Développement en Coopération (ORSTOM), 213, rue La Fayette, Paris, France ${ }^{1}$ Adresse actuelle : Centre de recherche sur les maladies virales émergentes, Université de Mahidol à Salaya, Thaïlande.

Correspondance : Dr. Ousmane Faye, laboratoire ORSTOM de Zoologie Médicale, Institut Pasteur, BP220, Dakar, Sénégal.

Tél : 2218234874 - Fax : 2218399210.

E-mail : Laurence.Lochouar@dakar.orstom.sn tion géographique du virus CCHF est vaste et s'étend à toute l'Afrique Sub-saharienne, au sud et au centre de l'Europe, et en Asie centrale (Hoogstraal, 1979). Ce virus est hautement pathogène pour l'homme mais des infections inapparentes existent (Watts et al., 1988). Les autres hôtes vertébrés ne développent pas de signes cliniques quand ils sont infectés. Les cycles de maintenance du virus CCHF sont complexes en raison de la variété des hôtes et des écosystèmes mis en jeu (Watts et al., 1988). Dans cette note nous rapportons les taux d'infection obtenus chez les imagos après une inoculation intra-coelomique. Les transmissions verticale (transovarienne et transtasiale) et horizontale (tique-vertébré) seront étudiées afin d'apprécier la place des trois espèces Amblyomma variegatum, Hyalomma marginatum rufipes et Hyalomma truncatum dans les cycles de maintenance et de transmission du virus CCHF au Sénégal. 


\section{MATÉRIEL ET MÉTHODE}

\section{SOUCHE VIRALE}

L a souche de virus CCHF HD 49199 isolée en 1988 à partir d'un cas mortel en Mauritanie a été utilisée au cours de nos expérimentations après un troisième passage sur des souriceaux nouveau-nés (SNN) (Gonzalez et al., 1990). Le titre était de 6 log $\mathrm{DL} 50 / \mathrm{ml}$. Cette souche est maintenue à $-70^{\circ} \mathrm{C}$ et les dilutions sont réalisées dans un milieu de Hanks.

\section{TIQues}

Au laboratoire, des colonies d'Amblyomma variegatum, de Hyalomma marginatum rufipes et de Hyalomma truncatum sont maintenues à partir de tiques femelles récoltées sur des bovins à Bandia au Sénégal $\left(14^{\circ} 35 \mathrm{~N}\right.$; $17^{\circ} 01 \mathrm{~W}$ ). Toutes les étapes du cycle de développement ont été étudiées en faisant gorger les différentes stases sur des lapins selon la méthode décrite par Cornet (1985).

\section{MÉTHODE D'INFECTION}

L'infection des imagos par voie intra-cœlomique est réalisée grâce à la technique de Lee \& Kemp (1970) modifiée par Huard et al. (1978). Elle consiste d'abord à préparer les seringues hypodermiques sur lesquelles on adapte des tubes capillaires préalablement effilés. Une dose d'inoculum à un titre de 3,5 log DL 50 est introduite en perçant délicatement la cuticule entre la première et la deuxième coxa sur la face ventrale. L'inoculation s'achève lorsque la tique étend complètement ses pattes préalablement recourbées. Environ 5 à $7 \mu \mathrm{l}$ d'inoculum sont introduits.

\section{PROCÉDURE (fig. 1)}

Pour chaque espèce, 150 imagos à jeun sont d'abord infectés par inoculation par voie intra-cœlomique avec la dose 3,5 log DL50/ 0,02 $\mathrm{ml}$. (Une partie de ces imagos sont ensuite testés entre J2 et J90 afin d'étudier la cinétique et la durée de l'infection). Après un temps d'incubation de 15 jours, des tiques infectées sont placées sur le dos des lapins rasés (deux couples par capsule) pour le repas sanguin. Un prélèvement sanguin est effectué avant la pose des tiques (J0) pour s'assurer que le lapin ne porte aucune trace du virus CCHF. Ces prélèvements se poursuivent jusqu'au

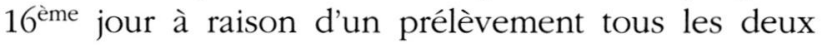
jours, temps à l'issue duquel les femelles gorgées se détachent de leur hôte. Les larves issues des femelles gorgées sont testées individuellement par écrasement sur lames avec masque de Téflon. Ces lames sont

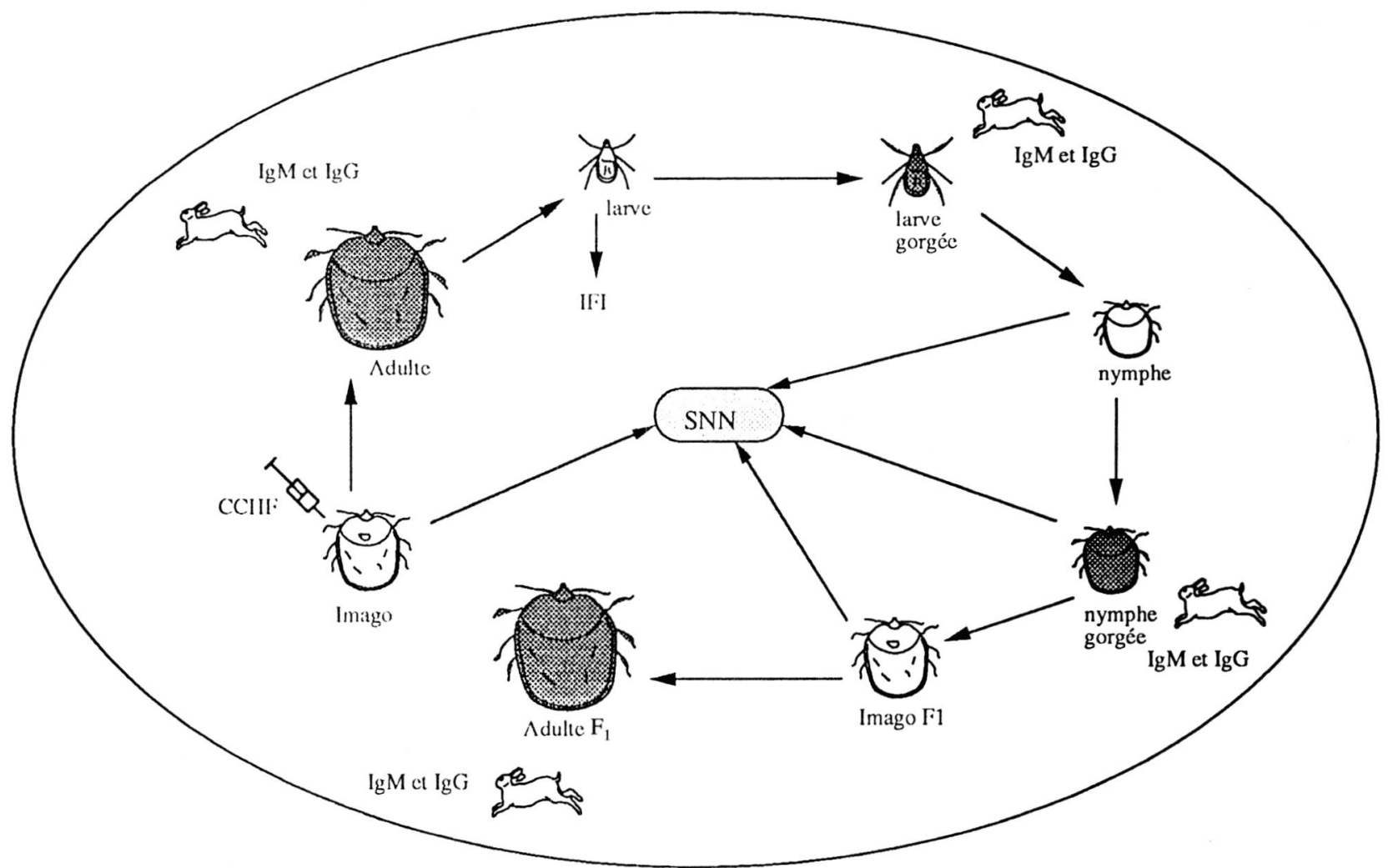

Fig. 1. - Protocole expérimental. IFI = immunofluorescence indirecte; $\mathrm{SNN}=$ souriceau nouveau-né; IgM et IgG = immunoglobulines des classes $M$ et $G$. 
ensuite colorées en immunofluorescence indirecte (IFI). Les nymphes et les imagos de la première génération (F1) sont d'abord testés par inoculation sur souriceaux nouveau-nés (SNN) et un contrôle est effectué par prélèvement des cerveaux de SNN malades et inoculation sur cultures cellulaires de type Vero. Chaque stase des générations mère et $\mathrm{F} 1$ est déposée sur des lapins pour étudier la transmission horizontale.

\section{Tests VIROlogiques et SÉROlOgiques}

Isolement : les isolements ont été faits sur SNN inoculés par voie intra-cérébrale (i.c.). Les suspensions virales utilisées ont été préparées à partir du broyat de tique dilué dans du milieu de Hanks.

Elisa : les sérums de lapin ont été testés en utilisant la technique ELISA de Niklasson et al., (1984), légèrement modifiée pour mettre en évidence les IgG antiCCHF. Dans cet ELISA direct les plaques de 96 cupules furent sensibilisées par des immuno-ascites de souris diluées au 1/1000 dans du carbonate de sodium (pH 9,6). Les antigènes témoins, négatif et spécifique du virus, puis les sérums à tester furent ajoutés. Des IgG de lapin associées à la péroxydase (Biosys) ont été utilisées pour révéler le complexe antigène-anticorps. Le substrat de la péroxydase est l'orthotoluidine. Les différences de densité optique (DO) entre le test et le contrôle sont mesurées à $450 \mathrm{~nm}$ en utilisant un lecteur automatique (Multiskan MCC 340). Les sérums sont considérés positifs si la différence de DO entre l'échantillon et le contrôle est supérieure à la moyenne des DO des témoins négatifs plus trois écart-types. Les immunoglobulines de la classe $\mathrm{M}$ sont détectées par immunocapture (Monath \& Nystrom, 1984). Les plaques sont sensibilisées avec du sérum anti chaine $\mu$ de lapin. Les sérums à tester suivis par les antigènes témoins, négatif et spécifique du virus CCHF, étaient alors associés. Les IgM dans le sérum à tester se fixent sur l'antigène. De l'immuno-ascite de souris anti CCHF a été ensuite ajoutée. La révélation a été faite avec de l'anti IgG de souris marqué à la péroxydase. La méthode du test et les critères sont les mêmes que ceux décrits pour les IgG. L'IFI a été réalisée selon la méthode classique décrite par Wulff \& Lange (1975). Une immuno-ascite de souris diluée au 1/100 ou un sérum de mouton infecté dilué au $1 / 16$ ont servi pour la mise en évidence de l'antigène et ont été révélés par un antisérum spécifique marqué à la fluoresceine. La lecture a été faite au microscope à fluorescence. Les cellules infectées étaient identifiées par la fluorescence spécifique d'inclusions intracytoplasmique et périnucléaire.

La réaction de transcription inverse et polymérisation en chaine (RT-PCR) pour la mise en évidence de l'ARN viral a été pratiquée sur les ARNs totaux obtenus par broyage d'une tique dans $800 \mu \mathrm{l}$ de tampon (Tris$\mathrm{HCl}, \mathrm{NaCl}$, EDTA, SDS $1 \%$, NP $401 \%$ ). L'ARN viral a été isolé après quatre extractions (deux phénol, une phénol-chloroforme et une chloroforme) et concentré par précipitation à l'acétate de sodium. La transcription inverse a été faite par incubation $30 \mathrm{mn}$ à $42^{\circ} \mathrm{C}$ d'un mélange de $1 \mu \mathrm{l}$ de l'oligonucléotide CSDR3 (GAC AAA TTC CCT GCA CCA) hybridé sur $(10 \mu \mathrm{l})$ d'ARN génomique dénaturé $\left(3 \mathrm{mn}\right.$ à $\left.95^{\circ} \mathrm{C}\right)$ dans un volume final de $20 \mu \mathrm{l}$ avec $0,5 \mu \mathrm{l}$ RNasine (Promega $\mathrm{N}$ $251 \mathrm{~A}), 4 \mu \mathrm{l}$ de tampon RT, $2 \mu \mathrm{l}$ d'NTP, $0,2 \mu \mathrm{l}$ de DTT et deux unités de transcriptase inverse (Gibco BRL ONH 118). La PCR a été réalisée dans un volume final de $100 \mu \mathrm{l}$ contenant du cDNA de transcription $(10 \mu \mathrm{l})$, un tampon taq $(10 \mu \mathrm{l}),(\mathrm{KCl} 50 \mathrm{mM}, \mathrm{MgCl} 21,5 \mathrm{mM})$, $2 \mu \mathrm{l}$ de d'NTP $(5 \mathrm{mM}), 1 \mu \mathrm{l}$ des amorces CSDF2 (5'TGG ACA CCT TCA CAA ACT C-3') et CSDR3, $1 \mu \mathrm{l}$ de taq polymérase (Amersham NK 9123) et $83 \mu \mathrm{l}$ de $\mathrm{H}_{2} \mathrm{O}$ stérile. Les conditions d'amplification génique consistent en 30 cycles de dénaturation $\left(95^{\circ} \mathrm{C}, 60 \mathrm{mn}\right)$, hybridation $\left(47^{\circ} \mathrm{C}, 30 \mathrm{mn}\right)$ et polymérisation $\left(72^{\circ} \mathrm{C}\right.$, $30 \mathrm{mn}$ ) suivis de $5 \mathrm{mn}$ à $72^{\circ} \mathrm{C}$. Le produit d'amplification (535 pb) a été isolé par migration électrophorétique de $10 \mu \mathrm{l}$ d'échantillon en gel d'agarose/TAE à $1,2 \%$ et révélé par coloration au bromure d'éthidium.

\section{RÉSULTATS}

TAUX D'INFECTION ET TITRE VIRAL

CHEZ LES TIQUES INFECTÉES

PAR VOIE INTRA-CELOMIQUE (tableau I)

hez Hyalomma marginatum rufipes : tous les
spécimens testés à partir du cinquième jour
post inoculation (pi) se sont révélés positifs avec un titre détectable sur les souriceaux. Le titre viral moyen chez les femelles qui est de 1,56 log DL 50/ $0,02 \mathrm{ml}$ à $\mathrm{J} 5$ pi croît jusqu'à $2,48 \log \mathrm{DL} 50 / 0,02 \mathrm{ml}$ à J10 et se stabilise à J15 avant de subir une légère diminution à J20 pi. Pour les mâles, une évolution à peu près semblable est observée avec des titres légèrement plus faibles : $1,49 \log \mathrm{DL} 50 / 0,02 \mathrm{ml}$ à $\mathrm{J} 5$ pi puis $2,17 \log$ DL 50/0,02 $\mathrm{ml}$ avant de subir une légère diminution au $20^{\text {ème }}$ jour.

\begin{tabular}{|c|c|c|c|c|c|c|}
\hline \multirow[b]{2}{*}{ Jpi } & \multicolumn{2}{|c|}{$\begin{array}{l}\text { Hyalomma } \\
\text { m. rufipes }\end{array}$} & \multicolumn{2}{|c|}{$\begin{array}{l}\text { Hyalomma } \\
\text { truncatum }\end{array}$} & \multicolumn{2}{|c|}{$\begin{array}{l}\text { Amblyomma } \\
\text { variegatum }\end{array}$} \\
\hline & Femelle & Mâle & Femelle & Mâle & Femelle & Mâle \\
\hline 5 & $1,56 \pm 0,13$ & $1,49 \pm 0,00$ & $1,43 \pm 0,00$ & $<1$ & $<1$ & $<1$ \\
\hline 10 & $2,48 \pm 0,08$ & $2,17 \pm 0,48$ & $1,84 \pm 0,22$ & 1 & $1,47 \pm 0,24$ & $<1$ \\
\hline 15 & $2,53 \pm 0,15$ & $2,22 \pm 0,26$ & $2,53 \pm 0,25$ & $1,90 \pm 0,15$ & $2,12 \pm 0,17$ & $2,25 \pm 0,09$ \\
\hline 20 & $2,17 \pm 0,25$ & $1,78 \pm 0,08$ & $1,86 \pm 0,33$ & $1,93 \pm 0,14$ & $2,26 \pm 0,05$ & $1,92 \pm 0,12$ \\
\hline
\end{tabular}

$\mathrm{Jpi}=$ jour post inoculation .

Titres en log DL 50/0,02 ml.

Tableau I. - Titres viraux après inoculation intra-cœlomique de trois Amblyommidae par le virus CCHF. 
Chez Hyalomma truncatum : à l'exception d'une tique mâle sur les 16 tiques testées où le titre était supposé faible, c'est-à-dire inférieur à $1 \log$ DL 50/0,02 ml, tous les spécimens testés à partir du cinquième jour ont montré une réplication effective du virus. Le titre moyen obtenu avec les femelles, 1,43 log DL 50/0,02 ml à $\mathrm{J} 5$ pi passe à $1,84 \log$ DL 50/0,02 ml au dixième jour, atteignant son maximum au $15^{\text {ème }}$ jour 2,53 log DL 50/ $0,02 \mathrm{ml}$. Les résultats obtenus avec les mâles suggèrent une réplication tardive avec $1 \log$ DL 50/0,02 ml au dixième jour pour atteindre un maximum 1,93 log DL $50 / 0,02 \mathrm{ml}$ au $20^{\text {ème }}$ jour.

Chez Amblyomma variegatum: La réplication semble être beaucoup plus lente aussi bien chez les mâles que chez

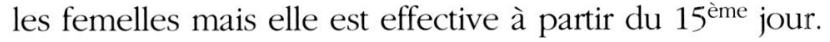

\section{DurÉE DE L'INFECTION CHEZ LES TIQUES INFECTÉES PAR VOIE INTRA-CCELOMIQUE}

Avec Hyalomma marginatum rufipes, l'observation s'est étalée sur une période de trois mois aussi bien pour les mâles que pour les femelles. Les résultats permettent de

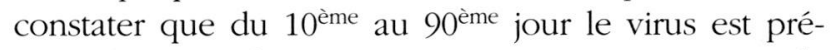
sent chez tous les spécimens testés avec un taux de $100 \%$ de mortalité souvent observé chez les souriceaux. Chez Hyalomma truncatum, un suivi d'un mois pour les mâles et de deux mois pour les femelles a été effectué. Les résultats obtenus montrent que cette espèce réplique rapidement le virus (toutes les tiques testées à partir du dixième jour sont positives) et qu'une fois infectées, elles conservent le virus au moins pendant toute la durée de nos observations correspondant à la période où les tiques étaient vivantes. Chez Amblyomma variegatum, un suivi de trois mois a été effectué tant pour les mâles que les femelles; la réplication n'est effective qu'entre le $15^{\text {ème }}$ et le $20^{\text {ème }}$ jour pi. Des taux de mortalité assez faibles et un contrôle sur cellules Vero négatif sont souvent observés durant les premiers jours de la multiplication.

Ainsi nous avons testé le virus par la technique PCR pendant les phases précoces de la multiplication (entre le $3^{\text {ème }}$ et le $15^{\text {ème }}$ jour $\mathrm{pi}$ ). Les spécimens positifs se caractérisent, après transcription inverse, amplification génomique et électrophorèse, par l'apparition d'une bande d'ADN de 535 paires de bases. Nos résultats montrent que l'ARN viral peut être détecté chez les Hyalomma dès le troisième jour pi et à partir du septième jour pi avec Amblyomma variegatum. Des bandes d'intensité différente liée au titres viraux apparaissent très nettement en fonction des jours post-inoculation.

\section{TRANSMISSIONS HORIZONTALE ET VERTICALE (tableau II)}

Hyalomma marginatum rufipes: compte tenu de la nature du cycle réalisé (dixène et monotrope), les imagos de la génération mère, les larves, les nymphes gorgées et les imagos de la génération $\mathrm{F}_{1}$ sont testés. Après 15 jours, l'infection des imagos était de $100 \%$ (15 positifs sur 15 testés). Sur 300 larves issues de trois pontes, 158 sont positives en IFI soit un taux de transmission transovarienne de $53 \%$. Sur dix nymphes gorgées et inoculées sur SNN, quatre sont positives après contrôle sur culture cellulaire de type Vero soit un taux de $40 \%$. Par contre le virus CCHF n'a pu être réisolé $(0 / 10)$ chez les imagos de la génération $F_{1}$ testés.

Une séroconversion est observée chez les trois lapins qui ont servi d'hôtes aux imagos inoculés. La cinétique d'apparition des anticorps révèle que les IgG et IgM apparaissent entre le sixième et le huitième jour post

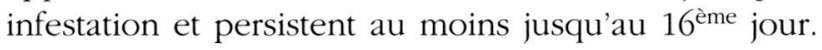
Les deux lapins, hôtes des stases larvaire puis nymphale ont eu des taux élevés d'IgG et d'IgM preuve d'une séroconversion. Cependant aucune trace du virus CCHF n'a été décelée avec les lapins $(0 / 2)$ qui ont servi d'hôte aux imagos de la génération $\mathrm{F}_{1}$.

Hyalomma truncatum : les imagos, les larves, les nymphes gorgées et les imagos de la génération $\mathrm{F}_{1}$ sont testés. Après 15 jours, l'infection des imagos était de $100 \%$ (15 positifs sur 15 testés). Sur 300 larves issues

\begin{tabular}{|c|c|c|c|c|c|c|}
\hline & \multicolumn{2}{|c|}{ Hyalomma m. rufipes } & \multicolumn{2}{|c|}{ Hyalomma truncatum } & \multicolumn{2}{|c|}{ Amblyomma variegatum } \\
\hline & $\begin{array}{c}\text { Test } \\
\text { virologique }\end{array}$ & $\begin{array}{c}\text { Test } \\
\text { sérologique }\end{array}$ & $\begin{array}{c}\text { Test } \\
\text { virologique }\end{array}$ & $\begin{array}{c}\text { Test } \\
\text { sérologique }\end{array}$ & $\begin{array}{c}\text { Test } \\
\text { virologique }\end{array}$ & $\begin{array}{c}\text { Test } \\
\text { sérologique }\end{array}$ \\
\hline & 1 & 1 & & & & \\
\hline Imago & $15 / 15(\mathrm{SNN})$ & $\{3 / 3\}$ & $15 / 15(\mathrm{SNN})$ & $\{3 / 3\}$ & $9 / 15(\mathrm{SNN})$ & $\{3 / 3\}$ \\
\hline Larve $\mathrm{F}_{1}$ & $158 / 300$ (IFI) & - & $150 / 300$ (IFI) & - & $24 / 200$ (IFI) & $\{2 / 3\}$ \\
\hline Nymphe $\mathrm{F}_{1}$ & - & & - & & $1 / 20(\mathrm{SNN})$ & \\
\hline Nymphe gorgée $F_{1}$ & $4 / 10(\mathrm{SNN})$ & $\{2 / 2\}$ & $3 / 10(\mathrm{SNN})$ & $\{3 / 3\}$ & $0 / 10(\mathrm{SNN})$ & $\{0 / 3\}$ \\
\hline Imago $\mathrm{F}_{1}$ & $0 / 10(\mathrm{SNN})$ & $\{0 / 2\}$ & $0 / 10(\mathrm{SNN})$ & $\{0 / 2\}$ & $0 / 10(\mathrm{SNN})$ & $\{0 / 2\}$ \\
\hline
\end{tabular}

1: nombre de positifs/nombre testé.

2 : test réalisé $:$ SNN $=$ souriceau nouveau-né $;$ IFI : immunofluorescence indirecte.

NB : Les valeurs entre crochets montrent les tests sérologiques réalisés sur les lapins hôtes des différentes stases. Tous les spécimens positifs présentaient des anticorps des classes IgG et IgM.

Tableau II. - Transmissions horizontale et verticale du virus CCHF chez trois Amblyommidae. 
de deux pontes, 150 sont positives en IFI soit un taux de transmission transovarienne de $50 \%$. Avec dix nymphes gorgées et inoculées sur SNN, trois sont positives après contrôle sur culture cellulaire de type Vero soit un taux de $30 \%$. Aucune infection n'a été obtenue avec les imagos de la génération $\mathrm{F}_{1}$.

Les résultats de la transmission horizontale sont identiques à ceux obtenus avec l'espèce $H$. m. rufipes avec une séroconversion chez les trois lapins qui ont servi d'hôtes aux imagos inoculés. La cinétique d'apparition des anticorps révèle que les IgG et IgM apparaissent entre le sixième et le huitième jour post infestation et persistent au moins jusqu'au 16 ème jour. Les trois hôtes des stases larvaire puis nymphale ont présenté des taux élevés d'IgG et d'IgM preuve d'une séroconversion. Cependant aucune trace du virus CCHF n'a été décelée avec les animaux $(0 / 2)$ qui ont servi d'hôtes aux imagos de la génération suivante.

Amblyomma variegatum : Le cycle de cette espèce est trixène. Ainsi les imagos de la génération mère, les larves, les nymphes, les nymphes gorgées et les imagos de la génération $\mathrm{F}_{1}$ sont testés. Après 15 jours, l'infection des imagos était de $60 \%$ (neuf positifs sur 15 testés). Sur 200 larves issues de deux pontes, 24 sont positives en IFI soit un taux de transmission transovarienne de $12 \%$. Après la métamorphose nymphale, 20 nymphes sont broyées individuellement puis inoculées chacune à un lot de dix SNN et un seul lot était positif soit un taux de $5 \%$. Avec dix nymphes gorgées et inoculées sur SNN, un taux de $0 \%$ a été observé. De même ce taux de $0 \%(0 / 10)$ a été obtenu avec les imagos de la génération $F_{1}$.

Une séroconversion a été observée chez les trois lapins qui ont servi d'hôtes pour les imagos inoculés (3/3). Par contre, deux des trois lapins ont fait une séroconversion après le repas larvaire. Cependant les animaux hôtes pour les stases nymphale puis imaginale de la génération $F_{1}$ n'ont montré aucune trace de séroconversion.

\section{DISCUSSION}

L a présence du virus chez les trois espèces testées et détectable sur le SNN montre que le virus CHF peut se répliquer efficacement chez les imagos inoculés par voie intra-coelomique. La multiplication virale après infection par voie intra-cœlomique avait déjà été observée chez Hyalomma truncatum (Okorie \& Fabiyi, 1980, Gonzalez et al., 1991), Rbipicephalus evertsi mimeticus et Amblyomma hebraeum (Shepherd et al., 1989) et paraît être la règle chez la plupart des Ixodides. Notre étude met en évidence une réplication plus faible pour Amblyomma variegatum par rapport à Hyalomma marginatum rufipes et Hyalomma truncatum. Cette différence de réplication est en accord avec les résultats de Shepherd et al., (1989) et Gonzalez et al., (1991). De même la réplication chez Hyalomma impeltatum, Hyalomma dromedarii, Hyalomma truncatum, et Rhipicephalus appendiculatus (Logan et al., 1990) montre un titre plus bas chez les espèces du genre Rhipicephalus. Ces observations font penser que des critères d'espèce d'ordre génétique pourraient permettre une meilleure réplication du virus chez les espèces du genre Hyalomma.

L'infection acquise après inoculation par voie intracœlomique persiste chez les imagos pendant toute la durée de nos observations (90 jours). Ces imagos infectés par voie intra-coelomique sont capables de transmettre le virus à leur hôte pendant le repas sanguin. La rapidité de l'infection chez les hôtes vertébrés suggère que les tiques transmettent le virus dès les premières heures de fixation. L'infection rapide des hôtes doit probablement se faire par la salive sécrétée lors de la phase préparatoire au cours de laquelle les tiques insèrent leurs pièces buccales et sécrètent un cément pour sécuriser leur fixation. De même les larves infectées de manière transovarienne transmettent le virus à leur hôte au cours du repas. Notre échec dans la détection des anticorps IgG et IgM chez les lapins après les repas des stases nymphale et imaginale de la génération $F_{1}$ constitue un argument en faveur de l'absence d'infection des glandes salivaires pendant la phase préparatoire du repas sanguin.

Les résultats observés montrent qu'au-delà de la persistance du virus observée pendant toute la durée de vie des tiques infectées, la transmission transovarienne peut assurer le passage du virus de la femelle infectée aux larves et aux nymphes via les œufs. Les taux de cette transmission sont élevés avec les espèces Hyalomma marginatum rufipes et Hyalomma truncatum, $53 \%$ et $50 \%$ respectivement. La transmission transtasiale (survie du virus pendant les métamorphoses larvaire et nymphale) suggère que l'infection virale pendant ces stases peut s'établir dans au moins un type de cellule qui ne subit pas d'histolyse. L'absence d'isolement du virus chez les imagos de la génération $F_{1}$ de toutes les espèces étudiées et pour les nymphes gorgées de Amblyomma variegatum suggère trois possibilités : 1) l'infection ne peut pas persister au-delà d'une génération; 2) le nombre de spécimens testés (dix) est trop faible; 3) les techniques ne sont pas assez fines pour la détection d'un taux viral faible.

Certains travaux sont en faveur de l'existence d'une transmission transovarienne du virus CCHF (Kodratenko, 1976), (Lee \& Kemp, 1970), (Wilson et al., 1991), tandis que les travaux de Shepherd et al., (1989) et Logan et al., (1990) sur les Ixodides concluent à une absence complète d'une transmission transovarienne. 
Finalement les résultats obtenus après écrasement individuel des larves sur lames, puis coloration en IFI, montrent l'existence d'une transmission transovarienne avec un taux de transmission élevé (53\%) chez Hyalomma marginatum rufipes et sont en faveur du taux minimal d'infection observé élevé avec cette espèce (Camicas et al., 1994).

Chaque stase infectée peut transmettre le virus à son hôte au cours du repas sanguin mais se limite à la génération $\mathrm{F}_{1}$ (Logan et al., 1989), (Gordon et al., 1993). Toutefois Zeller et al., (1994), en infectant des larves de Hyalomma marginatum rufipes sur le petit calao à bec rouge, réisolent le virus chez les larves $\mathrm{F}_{1}$. Cette non ou difficile persistance du virus pendant plusieurs générations montre que les tiques sont des vecteurs-réservoirs temporaires mais pas des réservoirs permanents du virus CCHF. Les vertébrés, en particulier les zébus, conservent donc un rôle central dans la circulation et le maintien de l'endémie dans la région biogéographique afro tropicale (Camicas et al., 1994).

\section{REMERCIEMENTS}

โ es auteurs remercient le Docteur Moreau, Directeur de l'Institut Pasteur de Dakar mais également Magueye Ndiaye, Carlos Fortes, Aïchatou Fall et Roughy Sylla pour leur aide technique. Ce travail a été financé par l'institut Français de Recherche Scientifique pour le Développement en Coopération (ORSTOM), par l'Institut Pasteur de Dakar et par la Communauté Économique Européenne.

\section{RÉFÉRENCES}

Camicas J.L., Cornet J.P., Gonzalez J.P., Wilson M.L., Adam F. \& Zeller H.G. La fièvre hémorragique de Crimée-Congo au Sénégal. Dernières données sur l'écologie du virus CCHF. Bulletin de la Société de Pathologie Exotique, 1994, $87,11-16$

Connet J.P. Contribution à l'étude de la biologie d'Amblyomma variegatum (Fab. 1784) dans la zone de transition Savane Forêt en RCA et de son rôle dans l'écologie de divers arbovirus. Thèse Doc. Sc. Orsay, France, 1985, 177 pp.

Gonzalez J.P., Le Guenno B, Gulllaud M. \& Wilson M.L. A fatal case of Crimean-Congo haemorrhagic fever in Mauritania: virological and serological observations suggest epidemic transmission. Transactions of The Royal Society of Tropical Medicine and Hygiene 1990, 84, 573-576.

Gonzalez J.P., Cornet J.P. Wilson M.L. \& Camicas J.L. Crimean-Congo hemorrhagic fever virus replication in adult Hyalomma truncatum and Amblyomma variegatum ticks. Research in Virology 1991, 142, 483-488.

Gordon, S.W., Linthicum K.J. \& Moulton J.R. Transmission of Crimean-Congo hemorragic fever virus in two species of Hyalomma ticks from infected adults to cofeeding immature formes. American Journal of Tropical Medicine and Hygiene, 1993, 48 (4), 576-580.

Hoogstraal H. The epidemiology of tick-borne Crimean Congo hemorrhagic fever in Asia, Europe and Africa. Journal of Medical Entomology, 1979, 15, 307-417.

Huard M., Cornet J.P., Germain M. \& Camicas J.L. Passage transovarien du virus Dugbe chez la tique Amblyomma variegatum (Fabricius). Bulletin de la Société de Pathologie Exotique, 1978, 7 (1), 19-22.

KODRATENKO V.F. Importance of ixodid tick in transmission and preservation of Crimean hemorragic fever agent in infection foci. Parasitology, 1976, 10, 297-302.

LEE V.H. \& KeMP G.E. Congo virus: experimental infection of Hyalomma rufipes and transmission to a calf. Bulletin of the Entomological Society of Nigeria, 1970, 2, 133-135.

Logan T.M., Linthicum K.J., Bailey C.L. Watts D.M., Dohm D.J. \& Moulton J.R. Replication of Crimean-Congo Hemorrhagic Fever virus in four species of Ixodid tick (Acari) infected experimentally. Journal of Medical Entomology, 1990, 27, 537-542.

Logan T.M., Linthicum K.J., Bailey C.L. Watts D.M. \& MoulTON J.R. Experimental transmission of Crimean-Congo Hemorrhagic Fever Virus by Hyalomma truncatum Koch. American Journal of Tropical Medicine and Hygiene, 1989, 40 (2), 207-212.

Monath T.P. \& Nystrom R.R. Detection of yellow fever virus in serum by enzyme-linked immunosorbent assay. American Journal of Tropical Medicine and Hygiene, 1984, 33, 151-157.

Niklasson B., Peters C.J., Grandiem M. \& Wood O. Detection of human immunoglobulin $G$ and $M$ antibodies to Rift valley fever by enzyme-linked immunosorbent assay. Journal of clinical Microbioloy, 1984, 19, 225-229.

OKorie T.G. \& FabiY A. The replication of Congo virus in Hyalomma rufipes Koch following intracoelomic innoculation. Veterinary Parasitology, 1980, 7, 369-374

Shepherd A.J., Swanepoel R., Cornel A.J. \& Mathee O. Experimental studies on the replication and transmission of Crimean-Congo Hemorrhagic Fever virus in some African tick species. American Journal of Tropical Medicine and Hygiene, 1989, 40 (3), 326-331

Watts D.M., Ksiazek T.G., Linthicum K.J. \& Hoogstraal H. Crimean-Congo hemorrhagic fever. in: The arboviruses : epidemiology and ecology, CRC Press, Monath T.P.(eds) Boca Raton, Fl., 1988, 177-222.

Wilson M.L., Gonzalez J.P, Cornet J.P. \& Camicas J.L. Transmission of Crimean-Congo hemorrhagic fever virus from experimentally infected sheep to Hyalomma truncatum ticks. Research in Virology, 1991, 142, 395-404.

WulfF H. \& LANGE J.V. Indirect immunofluorescence for the diagnosis of Lassa fever infection. Bulletin de l'Organisation Mondiale de la Santé, 1975, 52, 429-435.

Zeller H.G., Cornet J.P. \& Camicas J.L. Experimental transmission of Crimean-Congo hemorragic fever virus by west African wild ground-feeding bird to Hyalomma marginatum rufipes tick: American Journal of Tropical Medicine and Hygiene, 1994, 50 (5), 676-681.

Reçu le 24 août 1998 Accepté le 18 novembre 1998 